\title{
Artificial Intelligence: A Survey on Evolution and Future Trends
}

\author{
Maad M. Mijwil ${ }^{1}$, and Rana A. Abttan ${ }^{2}$ \\ ${ }^{1}$ Computer Techniques Engineering Department, Baghdad College of Economic Sciences University \\ Baghdad, Iraq \\ Email: mr.maad.alnaimiy [AT] baghdadcollege.edu.iq \\ ${ }^{2}$ Computer Techniques Engineering Department, Baghdad College of Economic Sciences University \\ Baghdad, Iraq \\ Email: rana.ali.abttan [AT] baghdadcollege.edu.iq
}

\begin{abstract}
Today, the world has heard a lot about artificial intelligence (AI) and its influence in accomplishing responsibilities, and it has become famous through films, series TV and social networking sites. Artificial Intelligence (AI) is a combination of algorithms and techniques developed by developers and programmers to build metal bodies that can work for centuries with individuals. Despite the interest of everyone in this topic and its spread significantly, most people do not have adequate knowledge and understanding of this science. This science is considered as one of the essential topics in computer sciences and engineering. In this article, it has been decided to write an overview on the topic of artificial intelligence and understand how its ideas started and spread universally. In addition, there is a review of Expert Systems, Artificial Neural Networks, Fuzzy Logic, and AI applications in the medical field and power systems, especially in investigating lung images of people with COVID-19. The idea presented in this article is that the future will soon come when humans and machines will merge into cyborgs or cybernetic creatures, and they will work together when completing tasks. This idea is described as transhumanism.
\end{abstract}

Keywords-Artificial Intelligence, Artificial Neural Networks, Expert System, Fuzzy Logic, COVID-19, Power Systems.

\section{INTRODUCTION}

First of all, the definition of artificial intelligence must be mentioned [1] [2]. In fact, there are many websites, articles and books that fully describe artificial intelligence, but in this article, another description of this science is given, that is, artificial intelligence refers to computers or computer-supported machines that have the ability to understand difficult algorithms. These abilities can build practices to implement higher-level processes, such as human personality, understanding, finding solutions, inferring meaning, and learning from past experience.

Actually, the idea of building non-life machines is an old idea and not modern as some think, as the age of this idea is approximately 4000 years. The world did not know the term artificial intelligence until the first references to artificial intelligence belong to the theory of computing of the British mathematician Alan Turing, who contributes to laying the foundations of the modern theory of computing and how to build and develop intelligent machines capable of processing data intelligently. In 1956 a workshop is held at Datmouth College, where the term artificial intelligence is used for the first time by the American computer scientist John McCarthy (Father of Artificial Intelligence), since that time and humanity has become day after day looking forward to knowing this science. In addition, the idea of creating and realising artificial intelligence has not been easy. The idea of its spread begins between 1974-1980, and this period is called "AI Winter," as many reports and newspapers have published criticism about the process of creating this science. Other scientists such as Allen Newell, Herbert Simon, and Claude Shannon are the first generation and founding fathers of the field of artificial intelligence as we know it today. Initial researches have focused on how to give machines the attribute of intelligence, the ability to analyse and reason in a similar way to humans.

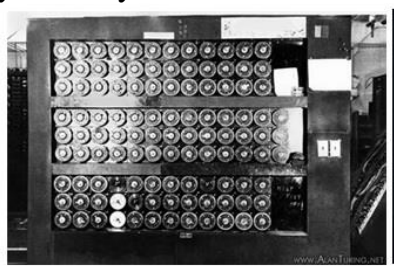

(a)

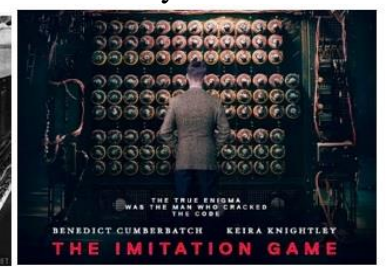

(b)

Figure 1: (a) The Enigma machine in original, (b) The Enigma machine in the film (downloaded from Goggle) 
In 2014 a film is produced starring the artist Benedict Cumberbatch (English actor) and entitled The Limitation Game, where this film tells the story of the British cryptanalyst Alan Turing, who helped a lot in deciphering the Enigma code used by the Germans during World War-II. The Turing machine is built, but it couldn't crack Nazi codes before resetting it every day and working on it is hard and tiring. Figure 1 exhibits Alan Turing with the Enigma machine in original and the cover of the Imitation Game film. Humanoid robots [3] are at the forefront of AI applications today. Interest in artificial intelligence and humanoid robots is growing day by day [4]. Nowadays, studies on this topic have increased dramatically to the point that robots can replace working people to reduce the workforce and activity in doing jobs and using them in many fields. At the end of 2020, an amazing dance video clip (robots dancing) has spread widely on social networking sites, especially Facebook, showing the Boston Academy, where four of its robot's dance performing dance moves designed entirely on the hit "Do you love me" by The Contours. This video has greatly aroused people's interest, a wonderful achievement in controlling robotics, and showed the ability and skills these robots have reached. Figure 2 displays some robotic snaps that are downloaded from Google. These robots can boogie down better than most humans.

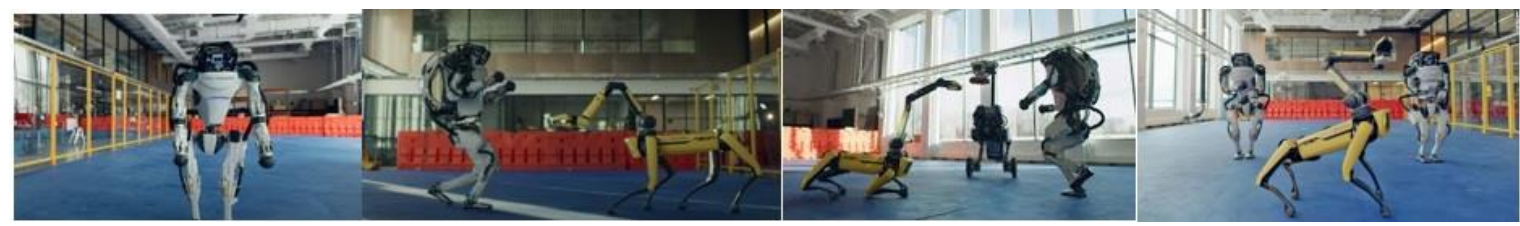

Figure 2: Some images of the Robot's dancing clip

In the 21 st century, films are a significant source of entertainment for the majority of people. People now prefer watching a 90-minute movie to reading a book of more than 400 pages, which basically contains the same amount of information. But what if I were to say that those 90 minutes could not only be entertaining but are also essential to kickstart your AI career. From massive machines to intelligent supercomputers, AI has been transforming human lives in untold ways - and it is safe to say it is only the beginning. With cinema creatively able to escape reality, today's AI films present this futuristic concept as part of our daily life now more than ever. Many beautiful films have appeared that brought the idea of artificial intelligence up, the most famous of these films are Ex Machina-2014, The Terminator-1984, and I, Robot-2004, and there are many films that show the future of artificial intelligence. Figure 3 shows posters of the most famous films (downloaded from Google).

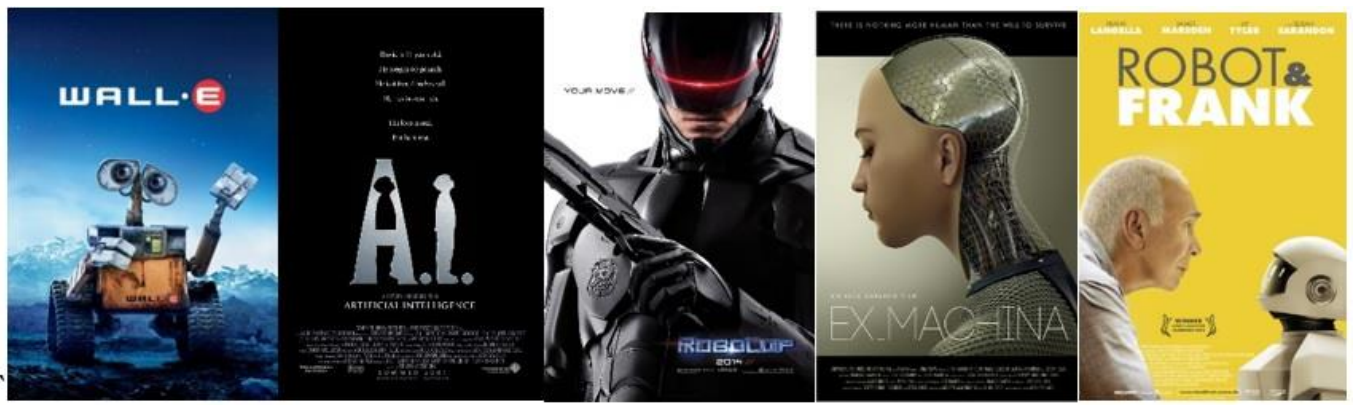

Figure 3: Five AI films

The field of artificial intelligence is a multidisciplinary field and is included in the field of study of different sciences such as computer engineering, electronics, control, mechatronics, programming, philosophy, astronomy and medicine. In this article, after presenting the description of artificial intelligence and its history, expert systems, artificial neural networks, and fuzzy logic are reviewed in detail, as well as the importance of artificial intelligence in medical fields is explored, especially in analysing lung images of people with COVID-19 infection, and AI in power systems. An article published by Singh et al. [5] represents an overview of artificial intelligence similar to the current article. We encourage anyone interested in artificial intelligence to read this article along with the current article.

\section{EXPERT SYSTEMS}

Expert systems [6][7] are methods with intelligent, computer-based, interactive and reliable specifications that can make decisions at any time and are distinguished by their ability to solve all complex problems. The decision-making stage is the most critical level of intelligence and human experience with these systems. Artificial intelligence simulates these processes with modern and advanced manners, and when we talk about expert systems, we mean solving problems that are more complex in a particular field. In other words, the artificial intelligence program includes enough stored knowledge to solve complex problems that humans cannot solve, and thus the role of a human expert comes to achieve 
them. However, these systems can also express and reason about ideas in a particular field of knowledge. Expert systems are the forerunners of today's artificial intelligence, machine learning and deep learning systems. On the other hand, expert systems are developed and determined by various traits, features and components such as a high level of expertise in thinking that provides competence, accuracy, imaginative problem solving and facing limitations. It also interacts at the right and appropriate time, that is, it interacts with the user in a very reasonable period of time. Expert systems are reliable methods that should not make any kind of errors or make decisions that are far from the problem. Also, these systems are characterized by being very flexible, and the mechanism of getting information is efficient. Also, an expert system is able to deal with complex problems and decisions at any time. In this way, it provides the most concise and compelling solution. In article published on your article library site by Priyali Sharma. He describes the components of expert systems, and we will talk about these components concisely [8]:

Knowledge Acquisition Subsystem: This is a knowledge engineer role. Since this is a skilful and time-consuming process, this often limits the design and operation of an expert system in a commercial environment. Knowledge Acquisition details are applied by an individual who has expertise in creating, adding to, or changing the knowledge base. Potential sources of knowledge involve human expert, investigation reports, textbooks, databases and the user's own experience. Experts base their judgments on quantitative and qualitative information. The system engineer has to translate all standard procedures into a formula suitable for the expert system. Gaining knowledge from experts is a significant point and often a bottleneck in creating the expert system professionally. Today, the knowledge engineer must have a significant role in interacting with one or more human experts in building the knowledge base.

Knowledge Base:It is considered as one of the most critical elements in an expert system because it includes solving any problem. It includes rules, facts, descriptions of things, etc. Besides, it is where the knowledge obtained from the expert is collected. The main obstacle facing any expert system is how knowledge is represented and how the knowledge base and information are stored. In other words, the knowledge base is stored in the collected data, and the information is stored in the knowledge base, which is necessary to understand and formulate the problem in order to be solved.

Interference Engine: Its main goal is to prepare and determine the mechanism for applying the knowledge in the knowledge base and depends on the facts and details presented by the user interface. Its faith is to draw conclusions about new facts and new information, which can be used to extract more and more new information in the future. This component is the brain of the expert system. Basically, this component is a computer program that executes the duties of processing the knowledge base to achieve a specific goal specified by the user, who interacts with the expert system via the user interface.

User Interface: The expert system has a language processor to communicate towards solving obstacles between the application administrator and the computer user, and it is favoured to communicate in a natural language. Sometimes it is better to use graphics to convey the idea. User interface technology (human computer interface) allows users to interact with the system. Some expert systems are distinguished by the fact that they can justify the outcomes reached, as well as the ability to describe why specific options are applied, or ignore and reject them.

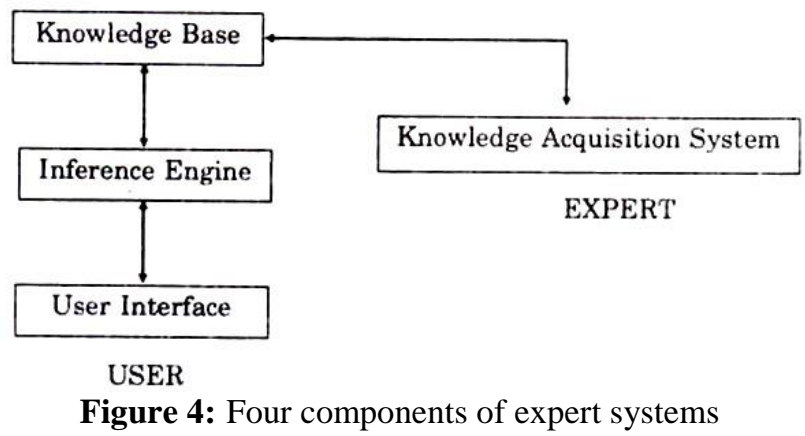

\section{ARTIFICIAL NEURAL NETWORKS}

Artificial neural networks (ANNs) [9] [10] are one of the widely used tools in artificial intelligence and machine learning. The idea of these tools is characterized by the idea of the work of the human brain aimed at continuously replicating the way in which humans learn to arrive at a correct approach to the goal of accomplishing a task. The ANNs consist of three layers (input/output and in most cases the hidden layer). They are also very excellent tools for finding very many patterns and, at the same time, being very complex for a human programmer to apply them in machine learning and build a correct structure in getting the task done without human intervention. These networks have been around since the 1940s and have another name, which is perceptron, and are now a large part of the science of artificial intelligence. ANNs have become popular after the arrival of the backpropagation technique, which allows any model of networks to adjust their hidden layers of neurons in situations where the result that the programmer codes are not applied. In short, if we have an application that recognizes animals, for instance, dogs, and when executing this application on an 
image consisting of a dog and a cat, it identifies only a cat, and it does not recognize or identify the dog. Figure 5 (a) presents the structure of neural networks in recognition of a dog's image through several images of animals.

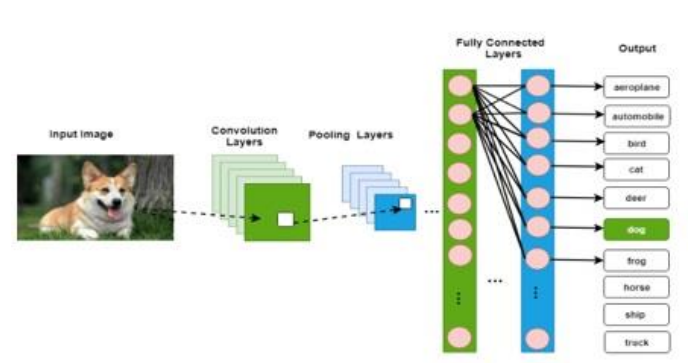

(a)

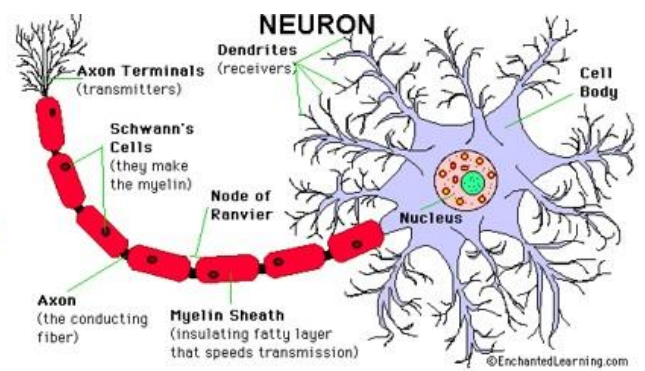

(b)

Figure 5: (a) Dog image recognition through Artificial neural networks [11], (b) The parts of single neuron in the human brain. [12]

Building connections between the many different processing elements in the system is the most significant characteristic of artificial neural networks, each analogous resembling a single neuron in the human brain. Each nerve cell takes many signals at once, then analyses them, inferred tasks, and then produces one signal that is sent as an input to another nerve cell. In fact, neurons are interconnected and coordinated in different layers. Furthermore, the hidden layer is sandwiched between the input and output layer, and it is one or more layers. These cells are simulated using a digital computer. Figure 5 (b) shows the parts of single neuron in the human brain. There are many types of neural networks, each of which has its own style of practice, implementation, and complexity levels. There is a type termed a Feedforward Neural Networks [13], and its function is to transmits information/data in one direction from an input to an output layer. The other type is Recurrent Neural Networks (RNNs) [14], they are transmitting information/data in multiple directions. Neural networks have a great experience to accomplish many complicated things, for example, language recognition; there is an article published in 2019 about the use of artificial neural networks in Turkish character (Türkçe karakterler) recognition written with a mouse [15]. There are also convolutional neural networks [16], Kohonen Self Organizing [17], Boltzmann machine networks [18], and Hopfield networks [19]. Picking the right network for a particular task depends on the type of data to be trained and the job done with it. Sometimes, it requires multiple methods to accomplish the task, such as a voice recognition task [20].

\section{FUZZY LOGIC}

Fuzzy logic [21][22] is one of the most powerful modern titles that the modern computer relies on because it depends in a currency on degrees of truth and does not depend on the usual method of Boolean logic (0 or 1). The idea of this topic is first introduced in the 1960s at Berkeley by Dr L. Zadeh of the University of California, USA. Zadeh's goal is to solve the problem of computer understanding of natural language, that is, to make the computer inclines towards activities, the implementation of any activities in life, any change in the usual and known method of computers that cannot be easily translated by 0 or 1 . Since that time, the idea of implementing robots has been capable of practising human life began without the use of old methods of building. Fuzzy logic is a closer idea to the human brain. In general, we collect data, and then a set of partial facts is established, which is further collected into higher facts which in turn, when certain thresholds are exceeded, cause some results such as motion interaction. Human-like capabilities and implementation in artificial intelligence to face any difficulty that a computer can solve without human intervention. Figure 7 illustrates the general architecture of the fuzzy logic systems.

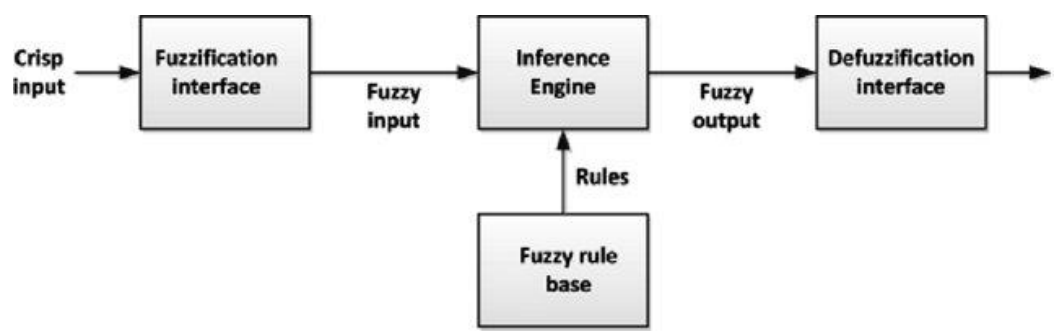

Figure 7: Architecture of a fuzzy logic system [23]

Fuzzy logic consists of several factors based on knowledge-based systems: the primary knowledge base, a rule base, and the inference system. Moreover, it is based on a defuzzification interface which is an elementary decision block in the system. In other words, the architecture of the fuzzy system is similar to that of the elementary decision-support systems. Fuzzy systems are characterized by ease of implementation, updating and adding new tasks in a currency due to 
their one-step inference performed. In general, fuzzy systems have the ability to draw conclusions through a set of class factors as well as use a graphical representation of them. Flexibility in fuzzy systems is the reason why these systems are used and applied in many fields, for example the medical field in analysing the image and patient information more accuracy.

At the end of this section, in a famous quote by L. Zadeh that "fuzzy logic is not the waves of the future". At present, there are many projects that depend on the principle of fuzzy logic, such as self-focusing cameras, washing machines that adjust themselves according to the extent of dirty clothes, tools for controlling car engines, colour-film developing systems, control systems in the subway and many tools that work according to the principle of fuzzy logic.

\section{AI IN MEDICAL FIELDS}

Technology and techniques are updating every day. Thanks to artificial intelligence, it is possible that machines can evaluate and analyse data to perform tasks the same way humans do properly. For this, techniques such as machine learning [24] or deep learning [25] are utilised in carrying out these tasks. In this way, the new knowledge being processed is incorporated based on the algorithms designed for this purpose. In medicine, these technologies and techniques are of particular importance as they allow processing and analysing medical data to improve health management in predicting and detecting diseases. In addition, artificial intelligence makes disease diagnosis easier and can improve patients' health problems. Thanks to the ability provided by artificial intelligence to detect certain health patterns, which will be very useful to speed up the solution of some medical problems that doctors and specialists need to speed up their eating and save the lives of patients. In short, today, artificial intelligence is the key to analysing and processing medical data thanks to its high processing capacity, as well as making medical diagnoses with greater accuracy and detecting diseases with small margins of error.

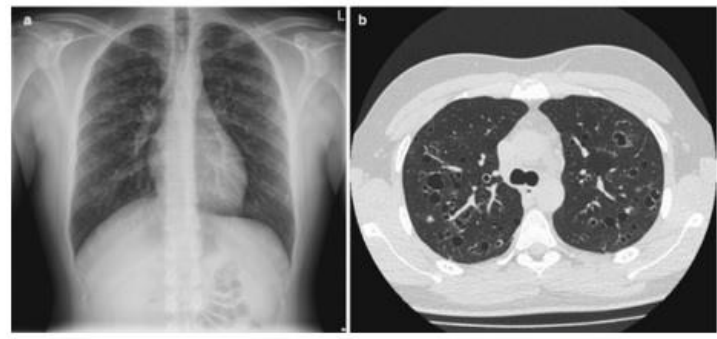

(a)

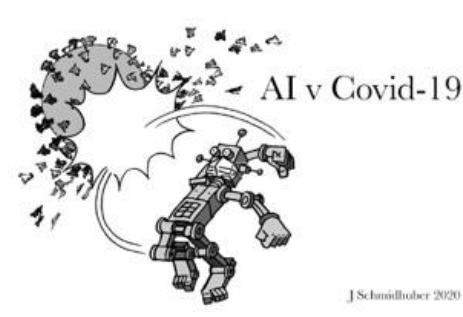

(b)

Figure 8: (a) X-rays and CT scan-images of infected persons with COVID-19, (b) Caricature image by Schmidhuber.

'In early 2020, researchers have begun experimenting with the use of artificial intelligence techniques in analysing X-ray images and CT-images for people with COVID-19 and experimenting with these techniques to detect the incidence of a disease, as there are many articles published in the analysis of these images and each paper has achieved a result. After reviewing a set of published articles, we have concluded that artificial intelligence has the ability to analyse the image and detect the disease with high accuracy. Artificial intelligence has recently proven its ability to track disease inside a human lung, detect infection rate, predict disease, and also distinguish between COVID-19 [26] and pneumonia among the infected. Besides, blood samples can be analysed to detect a pandemic. Figure 8.a presents X-rays and CT scan-images of infected persons [27]. In an article published by Jürgen Schmidhuber, he talks about how AI v COVID-19 [28]. He has published a caricature describing the power of artificial intelligence in destroying COVID-19 (see Figure 8.b). Artificial intelligence can grow mobile heath applications where smart devices like watches, mobile phones, cameras, and wearable devices can be employed for diagnosis, contact tracing, and efficient monitoring in COVID-19. Artificial intelligence can also be utilised to predict the chances of recovery or mortality in COVID-19 and implement daily updates, storage and trend analysis, and charting the course of treatment. Artificial intelligence has proven in recent years its strength in the medical society, as it can give an accurate and fast diagnosis and reduce the burden of working on doctors and specialists to identify ways to develop and discover new drugs in the detection of diseases as is happening now in the investigation of a vaccine to treat people with COVID-19 disease.

\section{AI IN POWER SYSTEMS}

Power systems [29] [30] are one of the parts of electrical engineering that deals with the generation, distribution and use of electrical energy in all areas of life. In the mid-1980s, saving power in many areas of energy systems has been difficult and unwanted. Nowadays, artificial intelligence has a major role in power systems such as economic load dispatch, load forecasting, optimisation of generation and scheduling, transmission capacity and optimal power flow, real and reactive power limits of generators, bus voltages and transformer taps, load demand in interconnected large power system and their protections etc. It also plays a role in reducing power systems' efforts and analysing them successfully in 
the output analysis. Compared with hydrocarbon extraction, the most powerful renewable energy sources such as solar and wind energy cannot be predicted as planned because they rely on meteorological factors beyond our control. In view of the short-term misprediction of the level of renewable energy production will quickly turn into a major supply difficulty, over time, as we inevitably move towards the energy future, renewable energy plays a pivotal role in it. This difficulty will become more and more serious, which will also make a big difference. However, there are reasons to be positive. Artificial intelligence is well suited to making renewable energy production predictable, making it more reliable, cleaner, and more affordable. Scientists have confirmed that artificial intelligence can use historical weather data blocks to predict future production levels more accurately. By analysing historical usage patterns, it can also play the same role in predicting future demand.

The current manufacturing technology utilised to make solar panels requires rare earth elements and a temperature of 2,000 degrees Celsius, which is very high and requires energy from fossil fuels to reach them. Although these technologies provide clean, renewable energy, each solar panel manufactured is still not emission-free. Artificial intelligence solves this problem by promoting research on developing and using new materials suitable for solar panels. The desired results can be obtained through trial and error, and thousands of individual tests may be required before significant progress is made. With artificial intelligence, many of the most complex experiments and responsibilities can be automatically executed and analysed, thus speeding up this vital process and providing us with a new generation of solar panels that can be used without causing significant environmental damage Under production.

\section{CONCLUSIONS}

In this study, it has been possible to cover a small part of artificial intelligence and some technologies and their application in medicine and energy systems. Through everything mentioned, artificial intelligence is still developing day by day, and new ideas and concepts arise about this great science. Today, artificial intelligence has become a big cycle on our planet. It has entered many fields such as space, military, industrial, electricity, renewable energy, medicine, engineering, media, and many other fields. By the year 2040, the authors expect that artificial intelligence will have a great role in controlling everything and will do what humans are currently doing in a large proportion, despite the great criticism about the idea that artificial intelligence is the new human being. Still, the world is developing and advancing, and artificial intelligence needs to accomplish its work very quickly. This is what this science aspires to. We may see the age of robots and people with some and in all fields, which is the era of transhumanism. In fact, there is a great amount of explanation about artificial intelligence, but it cannot be mentioned it in one article. We hope to write other articles in the future and other applications. We wish the world to live in peace and get rid of the COVID-19 pandemic.

\section{REFERENCES}

[1] Bezboruah T. and Bora A., "Artificial intelligence: The Technology, Challenges and Applications," Transactions on Machine Learning and Artificial Intelligence, Vol. 8, no.5, pp:44-51, August 2020. http://dx.doi.org/10.14738/tmlai.85.8956

[2] Shaw J., Rudzicz F., Jamieson T., and Goldfarb A., "Artificial Intelligence and the Implementation Challenge," Journal of medical Internet research, vol. 21, no. 7 e13659. 10 July 2019, http://doi.org/10.2196/13659

[3] Stasse O., Flayols T., "An Overview of Humanoid Robots Technologies," In: Venture G., Laumond JP., Watier B. (eds) Biomechanics of Anthropomorphic Systems. Springer Tracts in Advanced Robotics, vol 124, pp: 281-310, August 2018. Springer, Cham. https://doi.org/10.1007/978-3-319-93870-7_13

[4] Davenport T., Guha A., Grewal D., and Bressgott T., "How artificial intelligence will change the future of marketing," Journal of the Academy of Marketing Science-Springer, vol.48, pp:24-42, January 2020. https://doi.org/10.1007/s11747-019-00696-0

[5] Singh G., Mishra A., and Sagar D., "An Overview of Artificial Intelligence," SBIT Journal of Sciences and Technology, vol.2, no.1, pp:1-4, 2013.

[6] Abu-Nasser B. S., "Medical Expert Systems Survey," International Journal of Engineering and Information Systems (IJEAIS), vol. 1, no.7, pp:218-224, September 2017.

[7] Žarković M. and Stojković Z., Analysis of artificial intelligence expert systems for power transformer condition monitoring and diagnostics, Electric Power Systems Research-Elsevier, vol.149, pp:125-136, August 2017. https://doi.org/10.1016/j.epsr.2017.04.025

[8] Sharma P., “Top 4 Components of Expert System MIS," Article link: https://www.yourarticlelibrary.com/management/mis-management/top-4-components-of-expert-system-mis/70412

[9] Shahid N., Rappon T., and Berta W., "Applications of artificial neural networks in health care organizational decision-making: A scoping review," PLOS ONE, pp:1-22, February 2019. https://doi.org/10.1371/journal.pone.0212356 
[10] Negassi M., Suarez-Ibarrola R., Hein S., Miernik A., and Reiterer A., “Application of artifcial neural networks for automated analysis of cystoscopic images: a review of the current status and future prospects," World Journal of Urology, vol.38, pp:2349-2358, January 2020. https://doi.org/10.1007/s00345-019-03059-0

[11] Aamir M., Rahman Z., Abro W. A., Tahir M. and, Ahmed S. M., "An Optimized Architecture of Image Classification Using Convolutional Neural Network," International Journal of Image, Graphics and Signal Processing, vol.11, no.10, pp:30-39, October 2019. https://doi.org/10.5815/ijigsp.2019.10.05

[12] Label a Neuron, Brain Cells, The Brain, Article online (2018): https://www.enchantedlearning.com/subjects/anatomy/brain/Neuron.shtml

[13] Dixon M. F., Halperin I., and Bilokon P., "Feedforward Neural Networks," Machine Learning in Finance, pp 111166. July 2020. https://doi.org/10.1007/978-3-030-41068-1_4

[14] Sherstinsky A., "Fundamentals of Recurrent Neural Network (RNN) and Long Short-Term Memory (LSTM) network," Physica D: Nonlinear Phenomena, vol.404, March 2020. https://doi.org/10.1016/j.physd.2019.132306

[15] Al-Zubaidi E. A, Mijwil M. M., and Alsaadi A. S., "Two-Dimensional Optical Character Recognition of Mouse Drawn in Turkish Capital Letters Using Multi-Layer Perceptron Classification," Journal of Southwest Jiaotong University, vol.54, no.4, pp:1-6, August 2019. https://doi.org/10.35741/issn.0258-2724.54.4.4

[16] Yamashita R., Nishio M., Do R. K. G., and Togashi K., "Convolutional neural networks: an overview and application in radiology," Insights into Imaging, vol.9, no.4, pp:1-20, June 2018. https://doi.org/10.1007/s13244018-0639-9

[17] Audrain-Pontevia A. F., "Kohonen Self-Organizing Maps: A Neural Approach for Studying the Links Between Attributes and Overall Satisfaction in a Services Context," Kohonen Self-Organizing Maps, vol.19, pp:128-137, January 2006.

[18]Zhang P., Li S., and Zhou Y., “An Algorithm of Quantum Restricted Boltzmann Machine Network Based on Quantum Gates and Its Application,” Shock and Vibration, Vol. 2015, Article ID 756969, pp:1-7, September 2015. http://dx.doi.org/10.1155/2015/756969

[19] Du K. L., and M. N. S. Swamy, "Hopfield Networks, Simulated Annealing, and Chaotic Neural Networks.," In: Neural Networks and Statistical Learning. Springer, London. https://doi.org/10.1007/978-1-4471-5571-3_6

[20] Nichie A., and Mills G. A., "Voice Recognition Using Artificial Neural Networks and Gaussian Mixture Models," International Journal of Engineering Science and Technology, vol.5, no.5, pp:1120-1129, May 2013.

[21] Lee C. C., "Fuzzy logic in control systems: fuzzy logic controller," IEEE Transactions on Systems, Man, and Cybernetics, vol.20, no.2, pp:404-418, April 1990. https://doi.org/10.1109/21.52551

[22] Phuong N. H. and Kreinovich V., "Fuzzy logic and its applications in medicine," International Journal of Medical Informatics, vol.62, no.2-3, pp:165-173, July 2001, https://doi.org/10.1016/S1386-5056(01)00160-5

[23] Ibrahim D., “An Overview of Soft Computing," In Proceedings of International Conference on Application of Fuzzy Systems and Soft Computing, ICAFS 2016, 29-30 August 2016, Vienna, Austria. https://doi.org/10.1016/j.procs.2016.09.366

[24] Doupe P., Faghmous J., and Basu S., "Machine Learning for Health Services Researchers," Methodology, vol.22, no.7, pp:808-815, July 2019. https://doi.org/10.1016/j.jval.2019.02.012

[25] Ker J., Wang L., Rao J., and Lim T., "Deep Learning Applications in Medical Image Analysis," IEEE Access, vol.6, pp:9375 - 9389, December 2017. https://doi.org/10.1109/ACCESS.2017.2788044

[26] Wua Y., Chena C., and Chan Y., "The outbreak of COVID-19: An overview," Journal of the Chinese Medical Association, vol.83, no.3, pp:217-220. March 2020. https://doi.org/10.1097/JCMA.0000000000000270

[27] Desai S. R., Prosch H., and Galvin J. R., Chapter 4-Plain Film and HRCT Diagnosis of Interstitial Lung Disease, in book: Diseases of the Chest, Breast, Heart and Vessels 2019-2022: Diagnostic and Interventional Imaging Hodler J., Kubik-Huch R. A., von Schulthess G. K., (eds.), February 2019.

[28] Schmidhuber J., AI v Covid-19, April 2020, Article online: https://people.idsia.ch/ juergen/ai-covid.html

[29] Nath R. P. and Balaji V. N., "Artificial Intelligence in Power Systems," IOSR Journal of Computer Engineering, pp:1-7,2014. https://doi.org/10.23883/ijrter.2018.4234.eywih

[30] Anwar A. and Abttan R. A., "Multiple Fault Detection, Classification and Location in Electric Power Transmission Lines in Matlab Environment," International Journal of Engineering \& Technology, vol.7, no.4.16, pp:111-119, 2018. https://doi.org/10.14419/ijet.v7i4.16.21790 